\title{
Téoros
}

Revue de recherche en tourisme

\section{Tourisme d'affaires et libre-échange}

Les effets de l'ALENA

\section{Normand Cazelais}

Volume 15, numéro 3, automne 1996

Le tourisme d'affaires

URI : https://id.erudit.org/iderudit/1075063ar

DOI : https://doi.org/10.7202/1075063ar

Aller au sommaire du numéro

Éditeur(s)

Université du Québec à Montréal

ISSN

0712-8657 (imprimé)

1923-2705 (numérique)

Découvrir la revue

Citer cet article

Cazelais, N. (1996). Tourisme d'affaires et libre-échange : les effets de l'ALENA.

Téoros, 15(3), 32-33. https://doi.org/10.7202/1075063ar d'utilisation que vous pouvez consulter en ligne.

https://apropos.erudit.org/fr/usagers/politique-dutilisation/ 


\title{
TOURISME D'AFFAIRES ET LIBRE-ÉCHANGE
}

\author{
LES EFFETS DE L'ALENA
}

\author{
Normand Cazelais, journaliste et géographe \\ Hydro-Québec
}

Ilest difficile d'évaluer l'impact de l'ALENA, l'accord de libre-échange avec le Mexique et les Etats-Unis, sur le tourisme, d'autant plus qu'il n'est dejà pas aisé de cemer les effets qu'a eus en cette matière l'accord canado-américain entré en vigueur le ler janvier 1989. Faute de données claires et d'éndes foullées sur la question, on en est encore réduit a formuler hypothèses et suppositions.

On peut croire que l'accord de libreéchange tripartite devrait, dans la foulée du préédent, favoriser un accroissement des affaires et, par conséquent, du tourisme d'affaires. Bien sûr, comme dans le cas des voyageurs d'agrement, la TPS et la TVQ forment, la première dans l'ensemble du Canada et la seconde au Québec en particulier, une espece de ticket modérateur de nature à refroidir les ardeurs des Mexicains et Américains qui voudraient intensifier leurs activités au nord du 45 e parallèle. L'industrie dite des colloques et congrès, tout comme celle des voyages de motivation, en pátira-telle ou, au contraire, y trouvera-t-elle un souffle nouveau?

On sạit déjà qu'ont été modifiées les règles relatives à l'admission des gens d'affaires désireux d'effectuer des séjours temporaires dans les autres pays signataires. Les formalités de douane ont été assouplies et libéralisées pour certaines catégories d'activités (de nature internationale, liées à la culture, lá recherche et la conception, par exemple). L'effet se traduira-t-il par une augmentation des affaires des agences et voyagistes, tant émetteurs que réceptifs, spécialisés dans la planification et l'organisation de voyages d'affaires?

Un grand point d'interrogation à cet effet est lié à la configuration que présentera bientôt le transport aérien au Canada. Entrent en ligne l'Open Sky mais aussi l'avenir financier des deux grands transporteurs nationaux, Air Canada et surtout Canadien International, sans compter les percées et le profit qu'y trouveront les sociétés américaines entraînées dans la mouvance...

Le libre-echange peut être porteur de multiples bienfaits pour le développement du tourisme en Amérique du Nord, l'avenir le dira. Pour les besoins du présent texte, nous nous limiterons à jeter un regard plus attentif à deux aspects:

1) Les effets généraux sur les investissements et la main-d'oeuvre.

2) Les conditions faites aux gens d'affaires originaires au Canada voyageant aux Etats-Unis et au Mexique.

\section{LES EFFETS GENERAUX SUR LES INVESTISSEMENTS ET LA MAIN-D'OEUVRE}

L'ALENA contient des clauses précises sur le règlement des litiges, des dispositions sur les politiques d'achat gouvemementales et des accords latéraux sur l"environnement et la main-doeuvre, toutes choses qui concernent le tourisme au premier chef. L'entente définit également les principes de base de tous les aspects commerciaux et financiers dont celui des services: traitement égal, d'une part, pour tous les fournisseurs de services des pays signataires et privilégié, d"autre part, par rapport à ceux des autres pays, transparence des règles qui doivent être claires et accessibles à tous.

Dans un expose fait lors du IVe congrès de la Confédération panaméricaine des écoles hôtelières et de tourisme à Montréal en octobre 1994, Kenneth R. Johnston, diplomate canadien specialisé dans le commerce international, rappelait que la mondialisation des services, y compris celui du tourisme, s'observe partout sur la planète. La création, soulignait-il, de grands ensembles économiques soutenus par des ententes commetciales comme l'ALENA dont les dif" férentes clauses sont ad'une grande portée pour l'industrie touristiques qui a ainsi acces, sans restriction, à tous les produits offerts par les pays signataires grâce aे l'abolition des barrières commerciales. Soulignons notamment que, sauf certaines exceptions, toute entreprise peut offrir ses services dans un autre pays signataire sans avoir à y ćtablir de lieu d'affaires. Ce sont autant de facteurs qui peu- 
vent avoir des incidences directes sur le tourisme d'affaires.

Les dispositions ales plus importantes:, relevait-il, touchent les investissements: *De manière générale, les services touristiques faisant l'objet d'échanges sont fournis par des établissements situés à l'étranger (hôtels, restaurants, parcs thématiques établis dans un autre pays signataire, etc.). Dans la majorité des cas, il ne devra subsister aucune limitation des nouveaux investissements ou des acquisitions d'entreprises existantes. Les investisseurs pourront désormais être protégés contre les expropriations arbitraires par les gouvernements.;

Enfin. comme le stipulent ses diverses dispositions, l'ALENA facilite l'entrée, à titre temporaire - ce qui est important en matière de tourisme d'affaires — de cadres et d'administrateurs participant à la gestion d'un investissement dans un autre pays, de professionnels tels que directeurs d'hotel et autres responsables chargés de l'implantation de nouvelles entreprises.

\section{LES CONDITIONS FAITES AUX GENS D'AFFAIRES ORIGINAIRES DU CANADA VOYAGEANT AUX ÉTATS-UNIS ET AU MEXIQUE}

C'est le chapitre 16 dudit accord qui traite, de façon spécifique, des voyages des gens d'affaires entre les pays signataires. Selon les termes mèmes du ministère fédéral des Affaires étrangères et du Commerce international, les dispositions de ce chapitre acomplètent plutôt qu'elles ne remplacent les exigences de ces pays en matière de séjour temporairem et I'ALENA *n' influe en rien sur l'application des lois adoptées en matière d'immigration pour chacun des pays signataires afin de préserver la santé et la sécurité publiques, ainsi que la sécurité nationales.

Pour permettre de mieux se débrouiller dans les méandres et le clair-obscur des textes légaux, ce ministère a publiế une brochure complémentaire, Mouvements transfrontaliers des gens d'affaires en veriu de l'Accord de I'ALENA, qui présente, dans leurs grandes lignes, les critères et formalites régissant les voya- ges au Mexique et aux États-Unis des gens d'affaires de nationalité canadienne. Elle contient également des informations sur les droits exigibles et les formulaires à obtenir.

L'ALENA distingue quatre catégories de touristes d'affaires:

- les xhommes et femmes d'affaires en visitew, qui se livrent à des activités commerciales liées à la recherche et la conception, à la culture, à la fabrication et la production, à la commercialisation, à la vente et la distribution, au service apres-vente et aux services généraux;

- les uprofessionnelsw, qui exercent des activités professionnelles établies à I'Appendice 1603.D.I de I'ALENA, en vertu de contrats conclus avec des entreprises ou pour des employeurs établis au Mexique ou aux États-Unis:

- les apersonnes mutées à l'intérieur d'une sociétécw, à savoir des employés qui auront à remplir des fonctions de gestionnaires ou de cadres de direction ou encore des fonctions nécessitant des connaissances spécialisées au profit de ces entreprises ou de leurs succursales ou filiales établies aux États-Unis ou au Mexique;

- les anćgociants et investisseurs $\$$ appelés, entre autres, à diriger des commerces de produits ou de services avec le Mexique et les États-Unis, à diriger, développer ou gérer des entreprises nécessitant des capitaux étrangers.

La brochure" décrit ce quil en est pour chaque catégorie, tels les critères généraux d'admissibilité concernant les hommes et femmes d'affaires en visite au Mexique et aux Etats-Unis:

- avoir la citoyenneté canadienne;

- demander une autorisation de séjour pour affaires;

- exercer une activité commerciale d'envergure internationale:

* ne pas avoir l'intention de s'intégrer au marché du travail de ces pays;

- avoir une source de rémunération principale et un lieu d'affaires principal situés à l'extérieur des États-Unis et du Mexique;

- satisfaire, en matière d'immigration, les exigences américaines ou mexicaines qui régissent les séjours tempotaires.

\section{NOTE}

(1) Elle traite en outre des conjoints et personnes à charge qui accompagnent ces touristes d'affaires et des visas temporaires d'entrée aux États-Unis pour les personnes anon viseses par l'ALENA (antistes de spectacle, travailleurs non specialises, etc.). Elle explique ce qui se passe en cas de changement ou de renouvellement de statut.

\section{BIBLIOGRAPHIE}

- AFFAIRES ÉTRANGERES ET COMMERCE INTERNATIONAL CANADA (1994), Mouwements transfrontaliers des gens d'affaires en veriu de l'Accond de I"ALENA, Otawa.

- CAZELAIS, Normand, Errangers d"ict et dailleurs - Un tourisme à visage homain. Montréal, XYZ Éditeur, Tone I. 1993, Tome II. 1995.

- JOHNSTON. Kenneth R. The Globaization of Hotel and Tourism Services - NAFTA, Les Actes du Congres, IVe congrès CONPETH (Confédération panaméricaine des écoles hôtelières et de tourisme). Montrêal, 24-27 octobre 1994.

- RODRiguez WOOG, Manuel et Pablo ALVAREZ ICAZA L., Mexican Economy and Tourism in Relation to the Free Trade Agreement: Initial Results, 1995 Travel and Tourism Resarch Association Annual Conference, Acapulco, 10-14 juin 1995. 